\title{
DEFORMATION MECHANISMS IN NI-BASE DISK SUPERALLOYS AT HIGHER TEMPERATURES
}

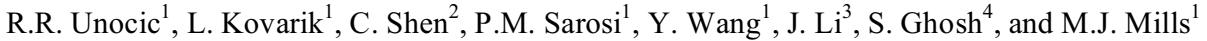 \\ ${ }^{1}$ The Ohio State University, Department of Materials Science and Engineering, Columbus, OH, 43210, USA \\ ${ }^{2}$ GE Global Research, Niskayuna, NY, 12309, USA \\ ${ }^{3}$ University of Pennsylvania, Department of Materials Science and Engineering, Philadelphia, PA 19104, USA \\ ${ }^{4}$ The Ohio State University, Department of Mechanical Engineering, Columbus, OH 43210, USA
}

Keywords: Ni-base Disk Superalloy, Creep, Deformation Mechanisms, Phase Field Modeling, Crystal Plasticity Modeling

\begin{abstract}
This paper presents results from a research initiative aimed at investigating high temperature creep deformation mechanisms in Ni-base superalloys through a combination of creep experiments, TEM deformation mechanism characterization, and state of the art modeling techniques. The effect of microstructure on dictating creep rate controlling deformation mechanisms was revealed for specimens with a bimodal $\gamma^{\prime}$ size distribution that possessed different secondary $\gamma^{\prime}$ size, tertiary $\gamma^{\prime}$ volume fraction, and $\gamma$ channel width spacing. It was found that the less creep resistant microstructure was the one with a greater secondary $\gamma^{\prime}$ size, wider $\gamma$ channel width, and higher volume fraction of tertiary $\gamma^{\prime}$. Deformation in this microstructure commences by way of $\mathrm{a} / 2<110>$ dislocations that are concentrated in the $\gamma$ matrix at lower strains, which then transition to a SISF precipitate shearing mode at larger strains. The more creep resistant microstructure possessed a finer $\gamma$ channel width spacing, which promoted $\mathrm{a} / 2<110>$ dislocation dissociation into $\mathrm{a} / 6<112>$ Shockley partials at lower strains and microtwinning at higher strains. Dislocation precipitate interaction was further explored using microscopic phase field modeling, which was able to capture key microstructural aspects that can favor dislocation dissociation and decorrelation since this appears to be a precursor to the microtwinning deformation mode. New viable diffusion pathways associated with the reordering processes in microtwinning have been explored at the atomistic level. All of the above activities have shed light onto the complex nature of creep deformation mechanisms at higher temperatures.
\end{abstract}

\section{Introduction}

Ni-base Superalloys are selected for use as components in the hot section of aircraft gas turbine engines since they possess the inherent capacity to retain strength and resistance to creep and fatigue at high homologous temperatures. They achieve such unique properties primarily through precipitation strengthening by coherent, ordered, $\mathrm{Ni}_{3} \mathrm{Al}$ based intermetallic $\gamma^{\prime}$ precipitates that are embedded in a disordered, solid solution, face centered cubic (fcc) $\gamma$ matrix. During high temperature creep deformation, the $\gamma^{\prime}$ precipitates play a vital role in restricting plastic deformation as high energy configurations such as antiphase domain boundaries (APB), complex stacking faults (CSF), superlattice intrinsic stacking faults (SISF), and superlattice extrinsic stacking faults (SESF) develop if the precipitates were to be sheared by a/ $2<110>$ type perfect matrix dislocations, $a / 3<112>$ super-Shockley partial dislocations, or $\mathrm{a} / 6<112>$ Shockley partial dislocations. There have been many detailed post mortem TEM characterization studies describing the deformation mechanisms responsible for creating such defect structures and implications they may have on controlling creep deformation for several different Ni-base superalloys [1-7].

Examples of the different deformation mechanisms that have been observed during creep deformation are: (1) Orowan looping and shearing of $\gamma^{\prime}$ particles by coupled $\mathrm{a} / 2<110>$ dislocations, (2) isolated faulting of the $\gamma^{\prime}$ particles, (3) thermally-activated microtwinning, (4) extended, continuous faulting of precipitates and matrix, and (5) dislocation climb by-pass at the highest temperatures. The preference for these mechanisms are dependent on factors such as alloy chemistry, the initial microstructure, stress, and temperature. Attempts to model the deformation response of these alloys within this temperature regime, without accounting for these detailed mechanism, will be of limited predictive capability.

The purpose of this paper is to summarize efforts under an Air Force funded program called MEANS (Modeling and Experiment for Affordable New Systems) that has been directed towards developing an improved, physics-based understanding of timedependent deformation in commercial Ni-base disk superalloys. With an emphasis on the integration of deformation experiments, detailed mechanism characterization, and state-of-the-art modeling techniques for the development of this fundamental understanding. While this effort has had special focus on Rene 104, the basic deformation mechanisms and developing understanding of them are expected to be of generic value to the $\gamma$-strengthened disk alloys.

\section{Materials and Experimental Procedure}

A newer generation advanced disk Ni-base superalloy ME3/Rene 104, that was recently co-developed by NASA, GE Aircraft Engines, and Pratt \& Whitney was the material chosen for this study $[8,9]$. The material was supplied in the form of a turbine disk forging that had been processed using a powder metallurgy route. A final supersolvus heat treatment procedure was applied to the forging, which resulted in the development of a bimodal distribution of $\gamma^{\prime}$ precipitates. In disk alloys, it is known that during a supersolvus heat treatment the rate at which the material cools from the $\gamma^{\prime}$ solvus temperature has a direct effect on the microstructural development of $\gamma^{\prime}$ precipitates, which will ultimately affect precipitate size scale, distribution, and morphology $[10,11]$. Due to the differences in cooling rate experienced throughout the cross section of the turbine disk following this heat treatment, different size scale and volume fraction of the coarser secondary and finer tertiary $\gamma^{\prime}$ precipitates have been developed in the outer rim and inner bore locations. 
Samples for microstructural analysis were extracted from these regions, mounted in conductive Bakelite, metallographically prepared using progressively finer $\mathrm{SiC}$ paper to 1200 grit then given a final $0.05 \mu \mathrm{m}$ colloidal silica polish. Samples were subsequently etched using a solution consisting of $2 \mathrm{ml} \mathrm{HF}, 30 \mathrm{ml}$ $\mathrm{HNO}_{3}$, and $50 \mathrm{ml}$ lactic acid. This solution preferentially etches away the $\gamma^{\prime}$ and accordingly the remnant structures were then imaged with a FEI Sirion SEM using an ultra-high resolution through lens detector. Afterwards, quantitative image analysis using Fovea Pro software was used to measure salient microstructural features (such as precipitate size, volume fraction, and $\gamma$ channel width spacing) that are known to have an influence on creep resistance.

Representative SEM images of the precipitate microstructure depicting the $\gamma^{\prime}$ size scale and bimodal distribution can be seen in Figure 1a-b for the bore and rim sections, respectively. In addition, principal microstructural features that were measured using quantitative image analysis techniques reveal an average secondary and tertiary $\gamma^{\prime}$ size of $350 \mathrm{~nm}$ and $30 \mathrm{~nm}$ for the bore microstructure and $180 \mathrm{~nm}$ and $30 \mathrm{~nm}$ for the rim microstructure. Due to the faster cooling rate experienced in the rim section, the average size of the secondary $\gamma^{\prime}$ are finer in size and the tertiary $\gamma^{\prime}$ are present to a much lesser degree when compared to the microstructure from the bore section, which experienced a slower cooling rate. Furthermore, the minimum separation distance between the coarser secondary $\gamma^{\prime}$ precipitates ( $\gamma$ channel width) was measured using a line intercept method, and was found to be larger for the bore microstructure as compared to the rim microstructure, on average $159.4 \pm 43.1 \mathrm{~nm}$ versus $63.5 \pm 10.2 \mathrm{~nm}$, respectively.

To investigate the influence of $\gamma^{\prime}$ precipitate morphology of these two different starting microstructures on creep behavior and to characterize the deformation substructure that evolves at different stages of deformation, constant load tensile creep experiments were performed at the same test conditions $\left(677^{\circ} \mathrm{C}, 724 \mathrm{MPa}\right.$, and interrupted at two levels of strain 0.05 and $1.0 \%$ ). Creep specimens, having a cylindrical gauge section measuring $4 \mathrm{~mm}$ in diameter and $16 \mathrm{~mm}$ in length, were extracted from the rim and bore regions and were machined using low stress grinding procedures by Metcut Research Inc. The amount of creep strain was measured using an averaging extensometer connected to two linear variable displacement transducers. Temperature was monitored and controlled using a type $\mathrm{K}$ thermocouple that was attached to the specimen.

Following creep testing, thin foils for TEM analysis were prepared by extracting sections from the gauge at a $45^{\circ}$ angle with respect to the tensile axis. This was done in order to view deformation activity along slip systems that experience maximum shear stress. The foils were mechanically thinned to a thickness of $100 \mu \mathrm{m}$ then slurry drilled to obtain $3 \mathrm{~mm}$ disks. Finally thinning was conducted on a Struers Tenupol 5 twin jet polishing unit using an electrolyte consisting of $60 \%$ methanol, $35 \%$ 2-nbutoxyethanol, and $5 \%$ perchloric acid at a temperature of $-45^{\circ} \mathrm{C}$ and with an applied voltage of $15 \mathrm{~V}$. Analysis of the post creep deformation structure was performed on a Phillips CM200 operating at $200 \mathrm{kV}$.

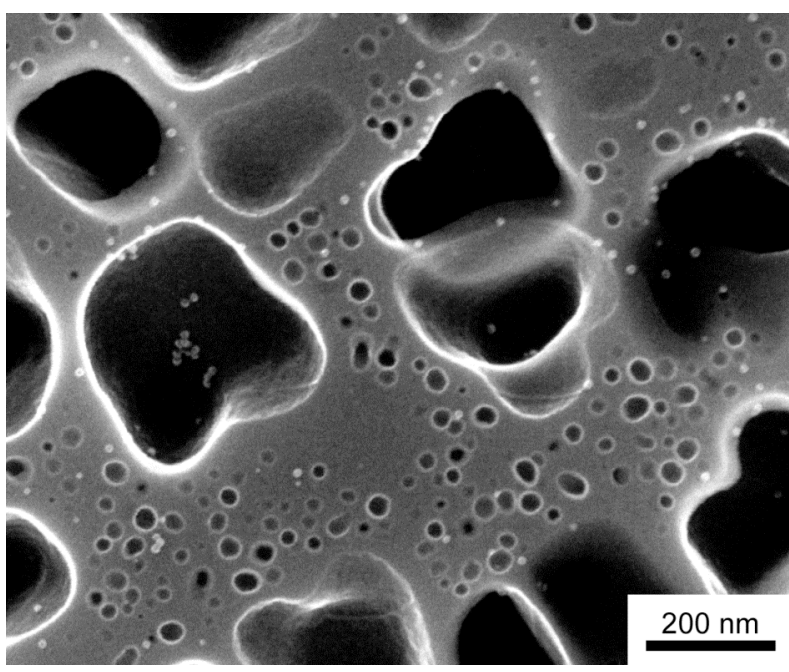

(a)

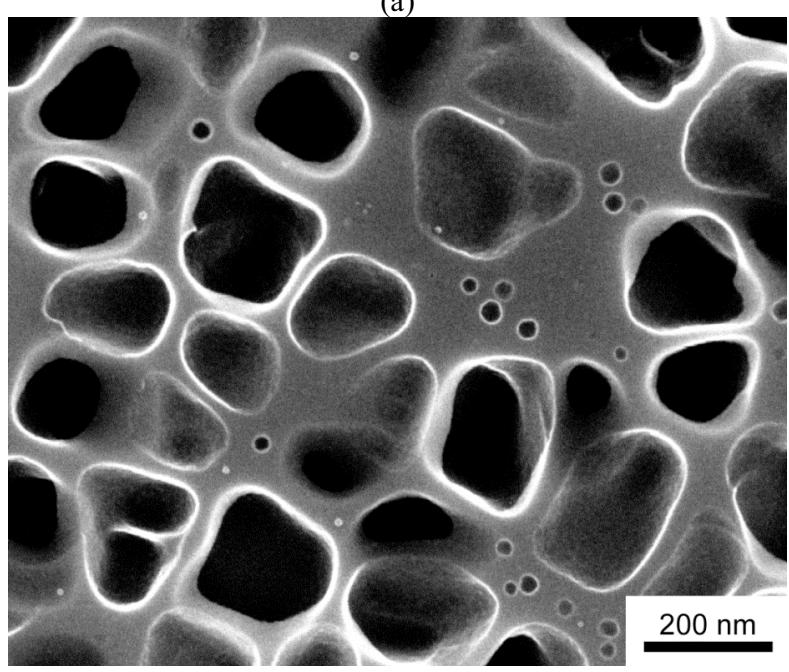

(b)

Figure 1. Representative SEM micrographs revealing the microstructural difference in $\gamma^{\prime}$ precipitate size, distribution, and morphology between the a) bore and b) rim sections of the turbine disk used in this study.

\section{Results}

\section{Creep Tests}

The results of creep testing are displayed in Figure 2 where creep strain is plotted vs. time for specimens carried out to slightly over $1.0 \%$ plastic strain. During the early stages of deformation both microstructures exhibit a normal primary creep transient period, which then transitions into a tertiary creep regime with creep rate continuously increasing with time. From multiple tests of these microstructures crept under the same test conditions but interrupted at different levels of strain, the normal primary transients and general form of the creep curve are reproducible. Since stress and temperature were identical for these test cases, it can easily be deduced that slight differences in the size scale of microstructural features can have a large effect of the creep deformation response of the material. 


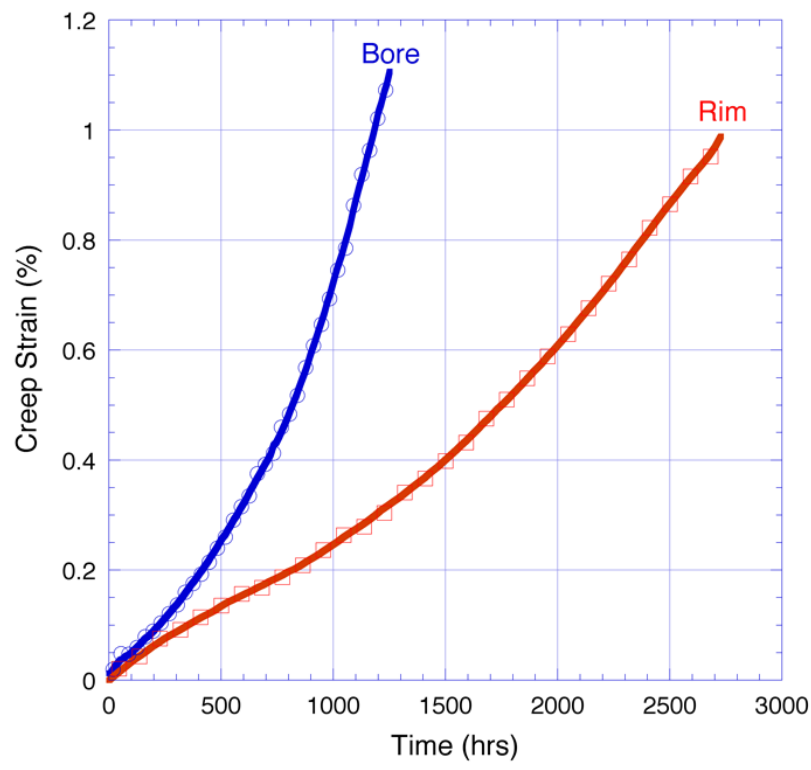

Figure 2. Experimental creep curves for the bore and rim microstructures tested at $724 \mathrm{MPa}$ and $677^{\circ} \mathrm{C}$ showing the difference in creep response.

\section{Deformation Mechanism Identification}

In order to expose the difference in creep behavior between the two different microstructures, a detailed TEM characterization study of the deformation structures that developed during the course of creep deformation was performed for specimens interrupted in the primary creep $(\sim 0.05 \%$ plastic strain $)$ and tertiary creep regimes $(\sim 1.0 \%$ plastic strain)

\section{Primary Creep Regime ( $0.05 \%$ Plastic Strain)}

TEM analysis of the creep deformation substructures for the bore and rim microstructures reveal that deformation is occurring predominately in the $\gamma$ matrix as little or no evidence of $\gamma^{\prime}$ precipitate shearing events were observed. However, there are clear differences in how plastic deformation has commenced for these two different microstructures. Beginning with the bore microstructure, Figure $3 \mathrm{a}$ displays the typical deformation substructure, which reveals a homogenous distribution of dislocations that are present in the $\gamma$ matrix. The Burgers vector of these dislocations were determined to be of the $a / 2<110>$ type through diffraction contrast TEM analysis.

The rim microstructure on the other hand, which has a finer spacing between the secondary $\gamma^{\prime}$ precipitates as well as a smaller volume fraction of tertiary $\gamma^{\prime}$ precipitates, deforms in a distinctly different manner. Again it is the matrix phase that deforms first at this early stage of creep deformation. Figure $3 b$ shows $a / 2<110>$ dislocations being emitted from an intergranular $\mathrm{M}_{23} \mathrm{C}_{6}$ carbide. Detailed dislocation analysis results show that these dislocations all have the same Burgers vector and that they have dissociated into Shockley partial dislocations that have a Burgers vector of the type $\mathrm{a} / 6<112>$. What sets this substructure apart from that seen in the bore microstructure is that the $a / 2<110>$ dislocations have dissociated into Shockley partial dislocations as evident from the intrinsic stacking faults present in the $\gamma$ matrix. The stacking faults appear in this image as extended grey regions of contrast in the wake of the leading and trailing Shockley partial dislocations.
The partial dislocations appear to have "percolated" between the secondary $\gamma^{\prime}$ precipitates, leaving them unsheared.

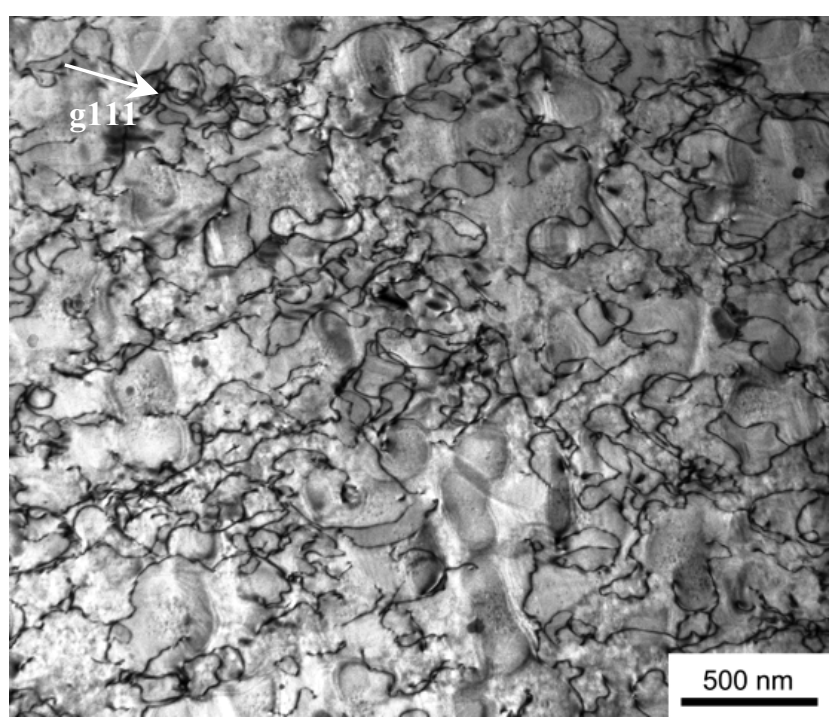

(a)

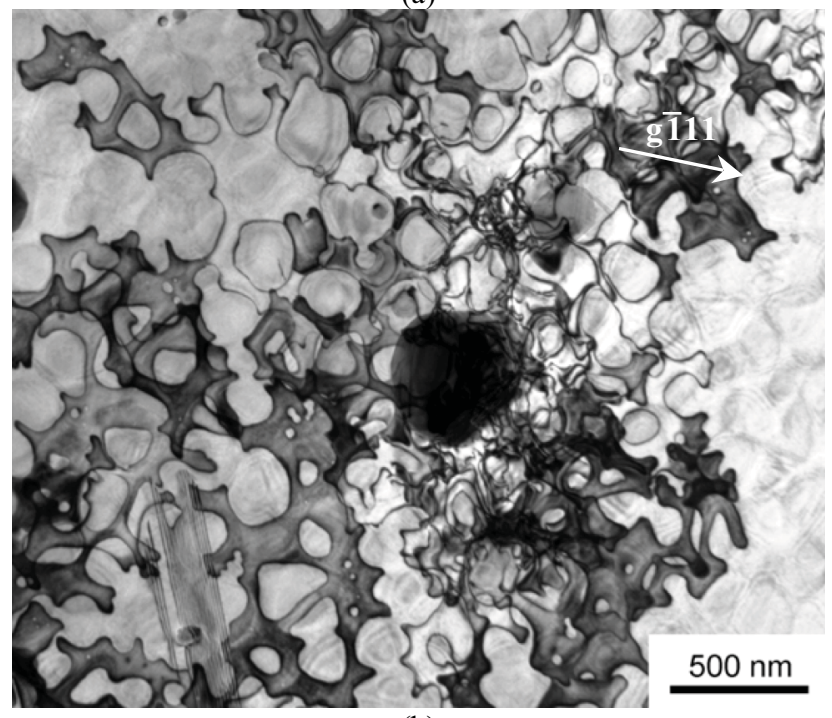

(b)

Figure 3. Post creep dislocation substructure interrupted after $0.05 \%$ strain at $677^{\circ} \mathrm{C}$ and $724 \mathrm{MPa}$ showing the difference in deformation mechanisms for the a) bore $(a / 2<110>$ matrix dislocation activity) and b) rim microstructures $(a / 2<110>$ dislocation dissociation into $\mathrm{a} / 6<112>$ Shockley partial dislocations bounding matrix intrinsic stacking faults).

\section{Tertiary Creep Regime ( $1.0 \%$ Plastic Strain)}

With an increase in plastic deformation there is also an increased amount of dislocation activity, as well as the appearance of distinctly different deformation mechanisms. Figure 4a shows a representative TEM micrograph of the dislocation structure for the bore microstructure crept to $1.0 \%$ plastic strain in $1175 \mathrm{hrs}$. It is evident that dislocations are again localized in the $\gamma$ matrix but to a much higher degree. Furthermore, there is also evidence of an additional deformation process that has commenced at this later stage of creep in which stacking faults have formed in the coarser secondary $\gamma^{\prime}$ precipitates, indicating that shearing of $\gamma^{\prime}$ is taking 
place. The faults were identified to be superlattice intrinsic stacking faults (SISF) based on complementary BF/DF imaging pairs. These single-layer stacking faults, that are active on multiple slip systems, were also observed to shear through $\gamma$ and $\gamma^{\prime}$ phases. In some cases it was possible to observe the dislocation responsible for shearing $\gamma^{\prime}$ within the particle itself, although the nature of the shearing partials have not yet been identified.

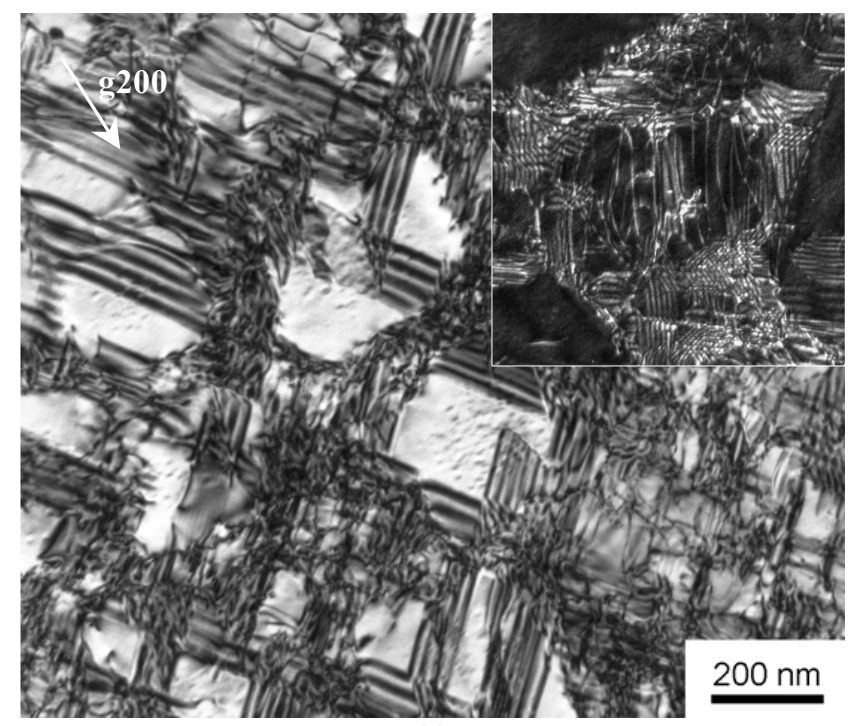

(a)

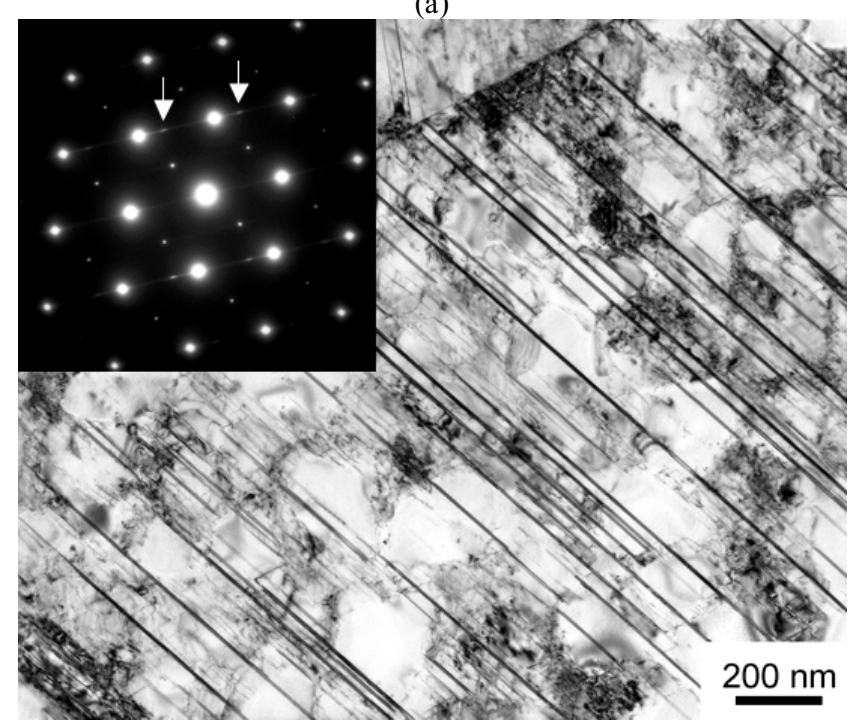

(b)

Figure 4. Post creep dislocation substructure interrupted after $1.0 \%$ strain at $677^{\circ} \mathrm{C}$ and $724 \mathrm{MPa}$ for the a) bore structure showing matrix dislocation filling by $\mathrm{a} / 2<110>$ dislocations (inset WB image) and superlattice stacking fault shear of the secondary $\gamma^{\prime}$. b) Rim structure crept to $0.98 \%$ showing microtwins oriented nearly edge on. Presence of twin reflections (as marked by arrows in the inset [011] ZA SADP) reveals that the highly planar deformation structures are microtwins.

For the rim microstructure that was crept to $0.98 \%$ plastic strain in 2725 hrs, a completely different deformation mechanism has developed. Microtwinning was found to be the dominant mode of deformation. Characteristic of the microtwinning mechanism are highly planar faulted structures that shear both precipitate and matrix by a/ $6<112>$ type Shockley partial dislocations on adjacent $\{111\}$ glide planes [7, 12]. Figure $4 b$ displays a BF TEM micrograph of the microtwins viewed nearly edge on with an inset selected area diffraction pattern directly on the [011] zone axis. The diffraction pattern shows fundamental reflections from the $\gamma$ matrix, superlattice reflections from the $\gamma^{\prime}$ precipitates, and twin reflections. The presence of these twin reflections indicates that these planar fault structures are microtwins. As can be seen in Figure $4 \mathrm{~b}$, many of the microtwins extend across entire grains. The remarkable observation that microtwinning is a principal creep process occurring at high temperature and low strain rate is the subject of further discussion below, and has motivated modeling activities at several different length scales.

\section{Discussion of Experimental Results}

To interpret and account for the differences in creep response it is important to focus on the main microstructural differences that set these structures apart from one another, namely: size scale of the secondary $\gamma^{\prime}$ precipitates, $\gamma$ channel width spacing, and volume fraction of tertiary $\gamma^{\prime}$ precipitates.

The early stages of creep in the bore microstructure is accommodated by $a / 2<110>$ dislocation activity. The spacing between the secondary $\gamma^{\prime}$ is relatively wide which allows for the $\mathrm{a} / 2<110>$ dislocations to pass between the secondary $\gamma^{\prime}$ with relative ease. There is however a high volume fraction of tertiary $\gamma^{\prime}$ precipitates that are present in the $\gamma$ channels between the secondary $\gamma^{\prime}$ precipitates that do provide resistance to dislocation motion and can ultimately affect the overall creep behavior as shown by Locq et al [13]. Shearing of these fine precipitates by $a / 2<110>$ dislocations will create anti-phase boundaries (APBs) in the precipitates. This process must be occurring since dislocation debris loops are not observed surrounding this fine population of particles. During prolonged time under creep conditions, the creep rate increase coincides with a large increase in the dislocation density within the $\gamma$ matrix. The enhanced dislocation activity in the matrix should be accommodated by precipitate shearing and SISF formation, which is occurring at higher strains.

Milligan et al has shown that SISF can be created as a result of a $\mathrm{a} / 2<\overline{1} 10>$ dissociation event that occurs at the $\gamma / \gamma^{\prime}$ interface and produces $\mathrm{a} / 3<\overline{2} 11>$ which shears the $\gamma^{\prime}$, creates a SISF and leaves behind a $a / 6<1 \overline{2} 1>$ Shockley partial at the interface during creep of single crystal PWA 1480 at $760^{\circ} \mathrm{C}$ [5]. Chen and Knowles have studied precipitate shearing and SISF formation as well. During creep of CMSX-4 at $750^{\circ} \mathrm{C}$ and $750 \mathrm{MPa}$ they have shown that the reaction and dissociation of two intersecting $\mathrm{a} / 2<110>$ matrix dislocations with different Burgers vectors can initiate $a / 3<112>$ slip which would create an SISF in the precipitate as it is sheared by the $\mathrm{a} / 3<112>$ super-Shockley partial dislocation [14].

Although direct evidence has not been obtained for this mechanism, it seems quite plausible that this may be occurring since there are many $a / 2<110>$ dislocations in the matrix that can intersect with one another to create this shearing configuration. Additionally, there are clearly multiple stacking fault configurations present, indicating that multiple slip systems have also been activated. The shearing of the secondary $\gamma^{\prime}$ may therefore be an important recovery process that helps relieve the 
strain hardening in the matrix, enabling continued dislocation activity in the matrix.

While the volume fraction of tertiary $\gamma^{\prime}$ precipitates may play a major role in affecting creep strength in the bore structure, it seems that the $\gamma$ channel width is controlling deformation for the rim structure at small strain, and may be a precursor to microtwinning at higher strain. The narrow channel widths, between the secondary $\gamma^{\prime}$ precipitates, appear to promote dislocation dissociation. Raujol et al have reported similar observations after creep deformation of disk alloy NR3 at $650 \mathrm{MPa}$ and $700^{\circ} \mathrm{C}$ [15]. Phase field dislocation dynamics simulations that support this hypothesis are discussed further in a following section.

In addition to the $\gamma$ channel width affecting dislocation dissociation and decorrelation of the Shockley partials, Suzuki segregation (which is the segregation of solute elements to stacking faults) might also aide in the dislocation dissociation process as the stacking energy may locally decrease, thereby increasing the separation distance between the leading and trailing partial dislocations. Segregation of solute elements that are known to lower the stacking fault energy, such as $\mathrm{Co}$ and $\mathrm{Cr}$ in $\mathrm{Ni}$, has been reported in Ni-base Superalloys [16]. Moreover, it is quite probable that Suzuki segregation can take place during high temperature creep conditions, which can facilitate diffusion of solute elements to stacking faults and assist in the dissociation and decorrelation process. Experimental energy dispersive X-ray spectroscopy (EDXS) line profiling of the stacking faults has provided preliminary evidence of segregation of $\mathrm{Co}$ and $\mathrm{Cr}$ to the matrix stacking faults, but ongoing microscopy investigations are directed at validating these findings.

It is envisioned that the dislocation dissociation and decorrelation process is a precursor to the microtwinning deformation mode since there is a transition to microtwinning during the later stages of creep for the rim microstructure. Microtwinning is another deformation mechanism that has been observed experimentally during high temperature creep in Ni-base superalloys [7, 12, 1721]. Kolbe first proposed that microtwins may form in a $\gamma / \gamma^{\prime}$ strengthened superalloy by the motion of paired identical a/ $6<112>$ Shockley partial dislocations operating on adjacent \{111\} glide planes shearing both $\gamma$ and $\gamma^{\prime}$ [17]. In the wake of the leading paired Shockley partials, a high energy, two-layer complex stacking fault (CSF) is created in the precipitate which must undergo atomic reordering in order to convert the pseudotwin structure to that of a true-twin, having the correct nearest neighbor atomic bonds for the ordered $\mathrm{L1}_{2}$ structure of the $\gamma^{\prime}$ phase. Further direct TEM evidence by Viswanathan et al [7, 12] in Rene 88DT has substantiated Kolbe's original hypotheses by confirming that the twinning partial dislocations are indeed identical Shockley partials.

For microtwinning, the rate controlling process is proposed to be the diffusion-mediated, atomic reordering that occurs in the wake of the Shockley partials which converts the high-energy, twolayer CSF to a low-energy, true-twin configuration [17, 22]. Karthikeyan et al recently developed a model in which the dislocation velocity was controlled by the rate of thermally activated reordering [22]. The model showed good agreement with the measured creep rates and provides a reasonable basis for understanding of creep in the rim microstructure of Rene 104. A more in depth analysis of the reordering process through atomistic modeling will be addressed in a later section.

At the present time it is not possible to provide a complete explanation for the differences in creep-rates for the two different microstructures since the rate limiting process associated with SISF formation in the bore structure is not presently identified. One possibility is that the reaction between $a / 2<110>$ matrix dislocations, which may be a precursor to SISF formation, is thermally activated. In this case, there may indeed be an activation barrier to the reaction. It is also possible that subsequent movement of the $\mathrm{a} / 3<112>$ super-Shockley partial into the secondary $\gamma^{\prime}$, thereby creating the SISF, may be a difficult thermally-activated process due to the complex core structure expected for this partial in the $\mathrm{L}_{2}$ structure [23].

\section{Modeling Activities Motivated by Experiments}

Insights from experimental creep testing and microscopic investigation of deformation mechanisms have motivated modeling at several length scales to shed additional light on the rate controlling creep processes. The microscopic investigation described above has revealed that the operative deformation mechanisms for the rim and bore microstructure are quite different at all stages of deformation that have been examined. The interaction of $a / 2<110>$ dislocation with precipitates and the various kinetic pathways associated with their dissociation have been explored using the so-called microscopic phase field (MPF) model [24]. The viable diffusion pathways of the reordering process associated with microtwinning have also been investigated at the atomic scale using $a b$ initio and empirical potential studies. At the highest level, a new crystal plasticity based formulation that attempts to incorporate these detailed insights is being developed. A brief synopsis of these modeling activities and initial results will now be discussed.

\section{Microscopic Phase Field Modeling}

Microscopic phase-field (MPF) modeling enables treatment of complex $\gamma^{\prime}$ shearing processes without any a priori assumptions about dislocation geometry, core structure, and formation of stacking faults [25]. The model is an extension of the Peierls model of a dislocation [26] with incorporation of the generalized stacking fault (GSF) energy [27] and 3D anisotropic microelasticity theory [28]. It utilizes a description of dislocations in terms of shear type inelastic strain fields, which combined with a formulation of pairwise elastic interaction between infinitesimal volume elements (i.e., a voxel-to-voxel interaction kernel), provides an efficient treatment for arbitrary dislocation geometries. The model stems from the phase field dislocation model [29] and its modification [30] with a focus at the atomic length scales. With $a b$ initio GSF energy, the MPF model is capable of treating quantitatively dislocation core structures. In addition, various planar faults are treated essentially as extended core structures and their formations are treated in a variational way.

As applied to the study of dislocation activity in the $\gamma / \gamma^{\prime}$ microstructure, the MPF model is advantageous in handling both arbitrary configurations of dislocations and microstructure, dislocation curvatures, dislocation dissociation and stacking fault formation, anisotropic elastic interactions among dislocations and between dislocations and microstructure, and taking into account the more physical diffuse dislocation cores instead of singular cores of Volterra type dislocation [31]. As a result of the MPF 
studies, an understanding of the critical microstructural features and deformation conditions that favor microtwinning versus other various stacking faulting modes has been obtained.

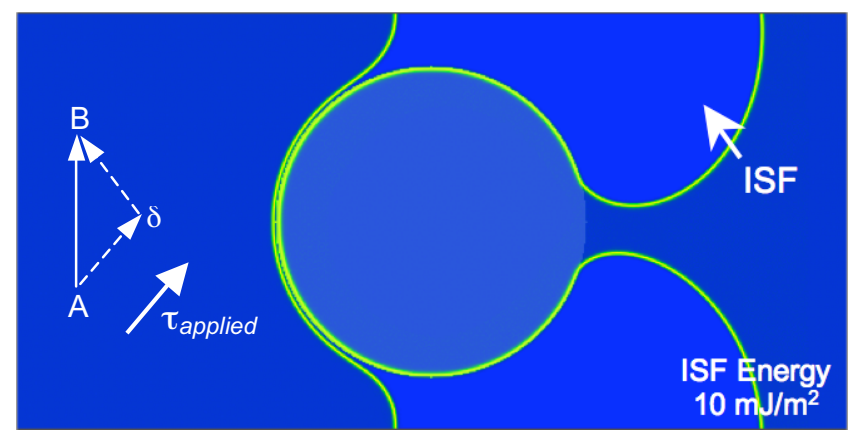

(a)

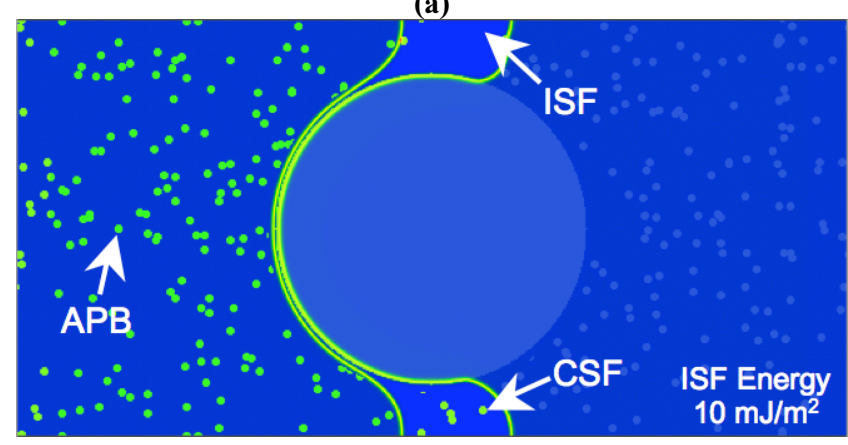

(b)

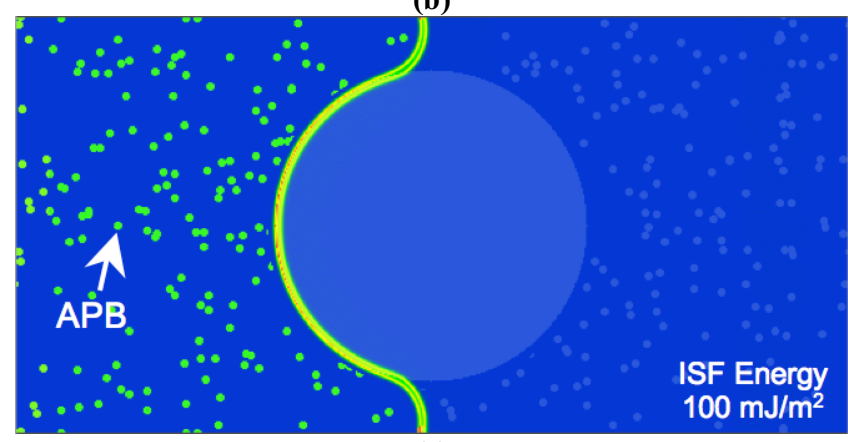

(c)

Figure 5. Phase field simulation of $\mathrm{a} / 2<110>$ dislocation (AB) interaction with a secondary $\gamma^{\prime}$ precipitate at an applied shear stress of $115 \mathrm{MPa}, 300 \mathrm{~nm} \gamma^{\prime}$ precipitate size, and $75 \mathrm{~nm} \gamma$ channel width spacing. The simulation shows the effect of tertiary $\gamma^{\prime}$ precipitates and stacking fault energy a) $10 \mathrm{~mJ} / \mathrm{m}^{2}$ ISF energy without tertiary $\gamma^{\prime}$ b) $10 \mathrm{~mJ} / \mathrm{m}^{2}$ ISF energy w/tertiary $\gamma^{\prime}$ and c) $100 \mathrm{~mJ} / \mathrm{m}^{2}$ ISF energy w/tertiary $\gamma^{\prime}$. (Periodic boundary conditions are applied in the vertical direction.)

A microscopic phase field simulation was developed to study the experimentally observed $\mathrm{a} / 2<110>$ dislocation dissociation into Shockley partials since it is believed that this process is a precursor to the microtwinning mechanism as described in a preceding section. The simulation is based upon a single screw oriented dislocation as it interacts with a secondary $\gamma^{\prime}$ precipitate population with a size (diameter) of $300 \mathrm{~nm}$ and $\gamma$ channel width spacing of $75 \mathrm{~nm}$. For this simulation, $\gamma^{\prime}$ precipitates were modeled to be spherical and the $\gamma$ channel width is defined as the spacing between the precipitates. A shear stress of $115 \mathrm{MPa}$ acting on the dislocations in a direction parallel to the Burgers vector of the

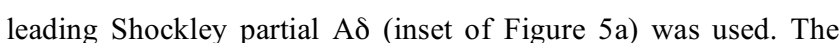
shear direction maximizes the force on the leading partial. Three configurations were initially chosen to show the feasibility of this model to capture the dissociation process and the results are presented in Figure 5a-c for the same total simulation time. The first two cases where the intrinsic stacking fault energy was fixed at $10 \mathrm{~mJ} / \mathrm{m}^{2}$ for cases without and with tertiary $\gamma^{\prime}$ precipitates are presented in Figure 5a-b, respectively.

In the phase field model, starting with the case without tertiary $\gamma^{\prime}$ precipitates, dissociation into Shockley partial dislocations occurs when the incoming dislocations come in contact with the secondary $\gamma^{\prime}$ precipitate and narrow $\gamma$ channel. As the applied stress is below the Orowan stress for the perfect dislocation (AB), it is sufficient to drive the leading partial $(A \delta)$ into the channel because of the smaller line tension (which is approximately proportional to $b^{2}$, where $b$ is the Burgers vector of the partial dislocation). Note the stress resolved on the trailing partial is only half that on the leading partial. The leading Shockley sweeps out and creates an intrinsic stacking fault in the matrix while the trailing partial remains at the junction. Similar results are observed when the simulation is run with a random distribution of tertiary $\gamma^{\prime}$ precipitates (Figure $5 \mathrm{~b}$ ), but in this case the presence of the tertiary $\gamma^{\prime}$ offers an additional impediment to dislocation motion as APBs are created in the wake of the passing a/2<110> dislocation, and CSFs are created in the tertiary $\gamma^{\prime}$ after being sheared by the leading Shockley partial dislocation.

Keeping the same configuration with tertiary $\gamma^{\prime}$, but increasing the matrix stacking fault energy 10 fold to $100 \mathrm{~mJ} / \mathrm{m}^{2}$ led to a quite different result as shown in Figure 5c. Under these circumstances, when the dislocation makes its way to the confined spacing of the $\gamma$ channel and due to the increase in matrix stacking fault energy, the dislocation does not dissociate into Shockley partials. Instead, the dislocation remains intact as a full $\mathrm{a} / 2<110>$ and was immobilized. From this simulation, it is clear that the matrix stacking fault energy is an important factor determining how the secondary $\gamma^{\prime}$ particles are by-passed, and dictating the critical stress for by-pass. These preliminary results of the phase field model demonstrate its effectiveness for performing parametric studies on the influence of microstructural size scale, changes in stacking fault energy (either by alloy chemistry or Suzuki segregation) and applied shear stress; detailed dependencies that are inefficient, if not impossible, to explore via experiment alone.

\section{Atomistic Reordering}

The novel reordering processes associated with the microtwinning mechanism will now be discussed further. It should be noted that this reordering process may also apply directly to other deformation mechanisms, such as the isolated faulting by superlattice extrinsic staking faults (SESFs) observed in Rene 88DT [7]. These reordering processes necessitate an atomic rearrangement in the wake of the partials to maintain the ordered $\mathrm{L}_{2}$ structure of the precipitates, which fundamentally explains the temperature and rate dependence during deformation in this regime.

As previously discussed, microtwins created by a/ $6<112>$ partial dislocations represent a configuration that contains energetically unfavorable Al-Al nearest neighbors. To signify the Al-Al nearest neighbor violation, the microtwins have been previously described as pseudo-twins. Kolbe showed that the pseudo-twins created by 
the passage of an even number of $a / 6<112>$ partial dislocations can reorder into a true-twin configuration by a diffusion process [17]. A schematic depiction of the reordering process for a twolayer microtwin is shown in Figure 6 . Two $a / 6<112>$ Shockley partial dislocations create a configuration with Al-Al nearest neighbor as depicted with the bonds. The bond violation is depicted only in the immediate vicinity of the partial dislocations because given sufficient time the configuration can reorder into a true-twin.

According to Kolbe, the reordering can be achieved by a two-step exchange process as shown in Figure 6. In the first exchange, $\mathrm{Ni}$ and $\mathrm{Al}$ atoms are replaced as indicated with arrow 1 . The second exchange involves the same $\mathrm{Ni}$ atom, but now it replaces another $\mathrm{Al}$ atom as shown with arrow 2. The three atoms involved in the exchange processes are from the (110) plane, which is perpendicular to the viewing direction. Depiction of the described process from a planer view of the central plane is shown in Figure $6 b-c$. The involved atoms are enclosed in a box. It is important to emphasize that the Kolbe reordering process does not involve an exchange between nearest neighbors, and as such cannot be accomplished directly by a vacancy. It should also be stated that Kolbe also proposed an alternative process, which does not involve the exchange between the nearest neighbors and thus has the same issue. An examination of the as-sheared and reordered configurations in Figure 6 reveals that there exists a much more direct way for the system to reorder from the pseudo-twin into the true-twin configuration. This direct way requires an exchange between $\mathrm{Al}$ and $\mathrm{Ni}$ atoms in the central plane of the microtwin as shown in Figure 7a. The involved $\mathrm{Ni}$ and $\mathrm{Al}$ atoms lie in a closed packed [110] direction, which is perpendicular to the $a / 6<112>$ Burgers vector. It should be noted that changing of $\mathrm{Al}$ and $\mathrm{Ni}$ in any other closed packed [110] directions would not accomplish reordering into a true-twin configuration.

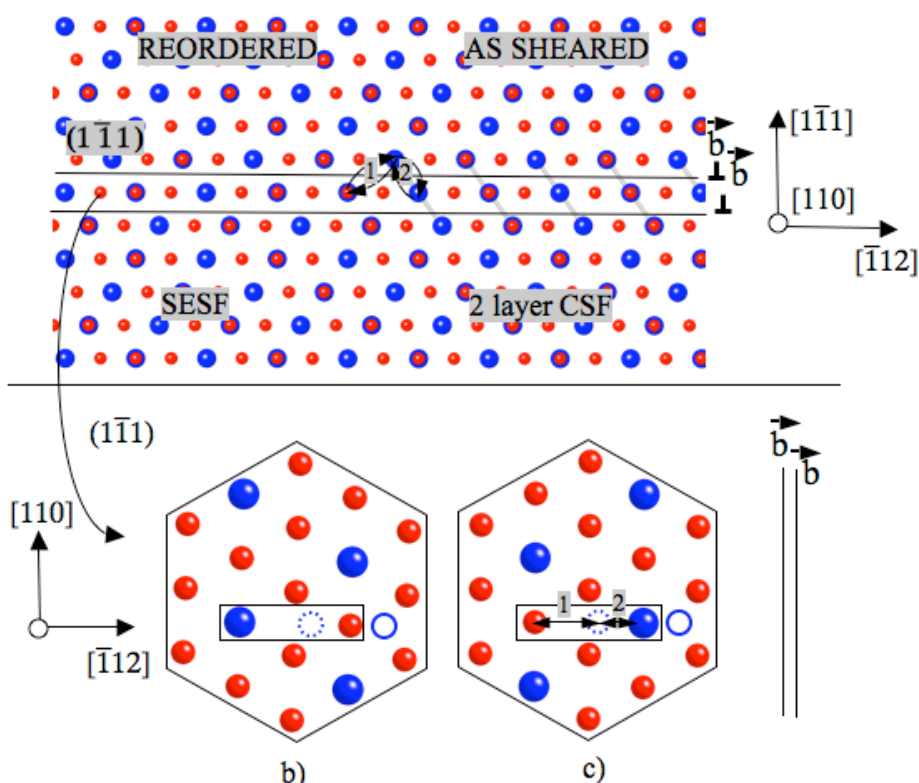

$\mathrm{Al}$ in a (11̄1) plane below

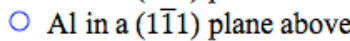

Figure 6. Two-layer microtwin that has been created by passage of two a/6 [112] partial dislocations on consecutive $\{111\}$ glide planes, as viewed along the [110] direction. (b). The central plane in the two-layer microtwin. Relative positions of Al atoms in the upper and lower adjacent plane is shown. (c) Identical part of the central plane after being reordered to a SESF.
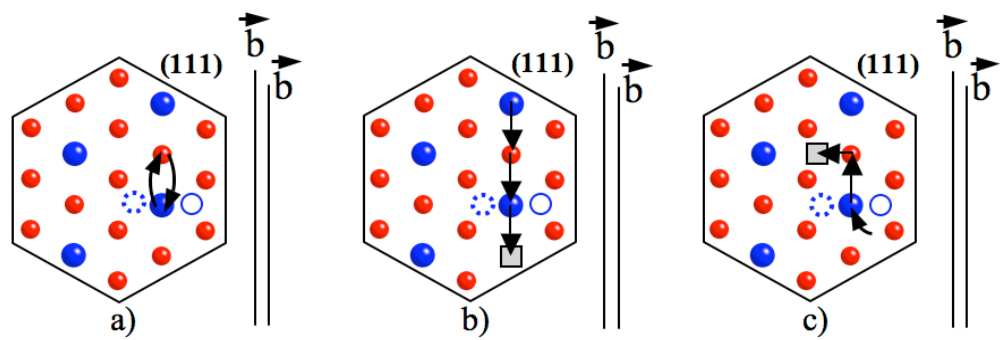

Figure 7. (a) Direct exchange of $\mathrm{Al}$ and Ni atoms that allow transformation of the "as-sheared" into a "true-twin" configuration. (b) An example of a linear movement of vacancy that enables an exchange between Al-Ni sites in an entire [110] column. (c) An example of less correlated case that allow single exchange between $\mathrm{Al}$ and $\mathrm{Ni}$ sites. 
The reordering of $\mathrm{Ni}$ and $\mathrm{Al}$ atoms in a microtwin is a vacancymediated diffusion process. One can easily envision several ways in which the vacancy movement can accomplish the swap between the Ni and Al sites. For example, in the simplest, most direct and highly correlated case, the vacancy would move in the [110] direction as shown Figure 7b. As the vacancy moves, the Al and $\mathrm{Ni}$ atoms in the column will be placed into the neighboring sites and thus assume positions that are consistent with a true-twin configuration.

One can readily envision many more viable-but less correlatedvacancy paths that will accomplish the exchange of the $\mathrm{Ni}$ and $\mathrm{Al}$ sites. One such alternate path is shown in Figure $7 \mathrm{c}$. In this less correlated case, the vacancy will enter the mixed column via $\mathrm{Ni}$ site, will accomplish one exchange between $\mathrm{Al}$ and $\mathrm{Ni}$ site and will exit from the mixed column. First principles calculations are currently being performed to determine the most likely atomvacancy movements, and calculate the corresponding energy barriers. The result of the calculations will be presented elsewhere [32], with the intent that the calculated energy barriers can ultimately be compared with the activation energy for creep under conditions for which the reordering process may be rate limiting.

\section{Dislocation-Based Crystal Plasticity FEM}

Crystal plasticity models remove dependencies on power law type empirical approaches, while facilitating tracking of dislocation evolution in each slip system through time. A recent dislocationdensity based crystal plasticity has been proposed for substructure-strengthened metals, for which geometrically necessary dislocations and statistically stored dislocations are considered [33]. While the basic framework of this model is quite flexible, for superalloys the model must be modified to incorporate the mechanism-specific attributes that are being identified through characterization and defect-level phase-field modeling. Karthikeyan et al [22] has proposed a "homogenized" model for microtwinning in which twinning partial velocity is postulated as a function of temperature, secondary and tertiary precipitate volume fraction.

However, in this initial model, twinning partial density evolution, nor transitions to other mechanisms, are not considered. For instance, as discussed above, microtwinning is initiated upon dissociation of mobile dislocations into Shockley partials when the precipitate structure and applied stress conditions are favorable. Assuming that a fraction $f$ of mobile dislocations decompose into these partials gives the density of twinning partials along each twin system as:

$$
\rho_{t p}{ }^{\alpha}=f(t) \cdot \rho_{M}{ }^{\alpha}
$$

We further assume that the secondary precipitates are the principal barriers that must be traversed by diffusion-mediate reordering, while the tertiary precipitates can be sheared athermally, resulting in a frictional stress. With these considerations, the effective resolved shear stress on twin system $\alpha$ can be obtained as:

$$
\tau_{\text {eff }}^{\alpha}=g\left(\tau^{\alpha}, \tau_{r e s}^{\alpha}, f_{2}, d_{2}, T\right)
$$

where $\tau^{\alpha}$ is the resolved shear stress, $\tau^{\alpha}{ }_{r e s}$ is the frictional shear stress due to tertiary precipitates and solutes, $f_{2}$ and $d_{2}$ are the volume fraction and size of secondary precipitates and $T$ is the dislocation line tension. By relating the average velocity of the dislocations to $\tau_{\text {eff }}^{\alpha}$ as:

$$
v_{p t}^{\alpha}=h\left(\tau_{e f f}{ }^{\alpha}, \rho_{p t}{ }^{\alpha}, \theta\right)
$$

an effective twin rate may be postulated from the classical Orowan equation as:

$$
\dot{\gamma}_{t w i n}^{\alpha}=\rho_{t p}^{\alpha} b_{t p}^{\alpha} v_{t p}^{\alpha}
$$

where $b_{t p}{ }^{\alpha}$ is the Burger's vector of the twinning partial dislocations. The detailed criteria dictating whether precipitates are sheared by isolated faulting or formation of continuous faults/microtwins is being studied in detail using the phase field modeling framework, and the results of these simulations will be presented elsewhere.

\section{Conclusion}

A combination of experimental creep testing, deformation mechanism identification, and computational modeling was used to study the creep deformation mechanisms and creep ratelimiting processes in the Ni-base disk superalloy ME3/R104. Differences in the initial $\gamma^{\prime}$ precipitate size scale, distribution, and channel width spacing were shown to have a large effect on the macroscopic response as well as the underlying deformation mechanisms. A microscopic phase field dislocation dynamics model was developed to investigate the microstructural aspects of dislocation dissociation and decorrelation, which apparently is a precursor to microtwinning at larger strains. The phase field simulations indicate that while the tertiary $\gamma^{\prime}$ play a role in hindering dislocation motion, it is the channel width and stacking fault energy that dictated whether dissociation occurs. These simulations offer considerable promise for parametrically studying the effect of applied stress, microstructure scale and fault energies on the deformation mechanisms. The key, thermally activated process associated with microtwinning appears to be diffusion-mediated reordering in the secondary $\gamma^{\prime}$ precipitates. The various possible diffusion pathways for reordering are being studied in detail using first principles modeling.

\section{Acknowledgements}

The authors would like to acknowledge support from the US Air Force Office of Scientific Research under the MEANS2 program Grant No. FA9550-05-1-0135.

\section{References}

[1] Kear BH, Oblak JM, Giamei AF. Stacking faults in gamma prime $\mathrm{Ni}_{3}(\mathrm{Al}, \mathrm{Ti})$ precipitation hardened nickel-base alloys. Metallurgical Transactions 1970;1:2477.

[2] Leverant GR, Kear BH. The mechanism of creep in gamma prime precipitation-hardened nickel-base alloys at intermediate temperatures. Metallurgical Transactions 1970;1:491.

[3] Mukherji D, Jiao F, Chen W, Wahi RP. Stacking fault formation in $\gamma^{\prime}$ phase during monotonic deformation of IN738LC at elevated temperatures. Acta Metallurgica et Materialia 1991;39:1515.

[4] Link T, Feller-Kniepmeier M. Shear mechanisms of the $\gamma^{\prime}$ phase in single-crystal superalloys and their relation to creep. 
Metallurgical Transactions A: Physical Metallurgy and Materials Science 1992;23A:99.

[5] Milligan WW, Antolovich SD. The mechanisms and temperature dependence of superlattice stacking fault formation in the single-crystal superalloy PWA 1480. Metallurgical Transactions A: Physical Metallurgy and Materials Science 1991;22A:2309.

[6] Decamps B, Raujol S, Coujou A, Pettinari-Sturmel F, Clement N, Locq D, Caron P. On the shearing mechanism of $\gamma^{\prime}$ precipitates by a single $(\mathrm{a} / 6)<112>$ Shockley partial in Ni-based superalloys. Philosophical Magazine 2004;84:91.

[7] Viswanathan GB, Sarosi PM, Henry MF, Whitis DD, Milligan WW, Mills MJ. Investigation of creep deformation mechanisms at intermediate temperatures in Rene 88 DT. Acta Materialia 2005;53:3041.

[8] Gabb TP, Telesman J, Kantzos PT, O'Connor K. Characterization of the temperature capabilities of advanced disk alloy ME3. NASA-211796, 2002.

[9] Gabb TP, Garg A, Ellis DL, O'Connor KM. Detailed microstructural characterization of the disk alloy ME3. NASA213066, 2004.

[10] Wlodek ST, Kelly M, Alden DA. Structure of Rene 88 DT. Superalloys 1996, Proceedings of the International Symposium on Superalloys, 8th, Champion, PA, Sept. 22-26,1996:129.

[11] Sarosi PM, Wang B, Simmons JP, Wang Y, Mills MJ. Formation of multimodal size distributions of $\gamma^{\prime}$ in a nickel-base superalloy during interrupted continuous cooling. Scripta Materialia 2007;57:767.

[12] Viswanathan GB, Karthikeyan S, Sarosi PM, Unocic RR, Mills MJ. Microtwinning during intermediate temperature creep of polycrystalline Ni-based superalloys: mechanisms and modeling. Philosophical Magazine 2006;86:4823.

[13] Locq D, Caron P, Raujol S, Pettinari-Sturmel F, Coujou A, Clement N. On the role of tertiary $\gamma^{\prime}$ precipitates in the creep behaviour at $700^{\circ}$ of a PM disk superalloy. Superalloys 2004, Proceedings of the International Symposium on Superalloys, 10th, Champion, PA, Sept. 19-23,2004:179.

[14] Chen QZ, Knowles DM. Mechanism of $<112>/ 3$ slip initiation and anisotropy of $\gamma^{\prime}$ phase in CMSX-4 during creep at $750^{\circ} \mathrm{C}$ and $750 \mathrm{MPa}$. Materials Science \& Engineering, A: Structural Materials: Properties, Microstructure and Processing 2003;A356:352.

[15] Raujol S, Benyoucef M, Locq D, Caron P, Pettinari F, Clement N, Coujou A. Decorrelated movements of Shockley partial dislocations in the $\gamma$-phase channels of nickel-based superalloys at intermediate temperature. Philosophical Magazine 2006;86:1189.

[16] Leverant GR, Kear BH, Oblak JM. The influence of matrix stacking fault energy on creep deformation modes in $\gamma^{\prime}$ precipitation-hardened nickel-base alloys. Metallurgical Transactions 1971;2:2305.

[17] Kolbe M. The high temperature decrease of the critical resolved shear stress in nickel-base superalloys. Materials Science \& Engineering, A: Structural Materials: Properties, Microstructure and Processing 2001;A319-321:383.

[18] Legros M, Clement N, Caron P, Coujou A. In-situ observation of deformation micromechanisms in a rafted $\gamma / \gamma^{\prime}$ superalloy at $850^{\circ} \mathrm{C}$. Materials Science \& Engineering, A: Structural Materials: Properties, Microstructure and Processing 2002;A337:160.

[19] Knowles DM, Chen QZ. Superlattice stacking fault formation and twinning during creep in $\gamma / \gamma^{\prime}$ single crystal superalloy CMSX-4. Materials Science \& Engineering, A:
Structural Materials: Properties, Microstructure and Processing 2003;A340:88.

[20] Ardakani MG, McLean M, Shollock BA. Twin formation during creep in single crystals of nickel-based superalloys. Acta Materialia 1999;47:2593.

[21] Unocic RR, Mills MJ. Unpublished Research. 2008.

[22] Karthikeyan S, Unocic RR, Sarosi PM, Viswanathan GB, Whitis DD, Mills MJ. Modeling microtwinning during creep in Ni-based superalloys. Scripta Materialia 2006;54:1157.

[23] Sun YQ, Hazzledine PM. Geometry of dislocation glide in $\mathrm{L1}_{2} \gamma^{\prime}$-phase. In: Nabarro FRN, Duesbery MS, editors. Dislocation in Solids, vol. 10. Amsterdam: Elsevier, 1996. p.27.

[24] Shen C, Li J, Wang Y. Manuscript in preparation. 2008.

[25] Shen C, Li J, Mills MJ, Wang Y. Modeling Shearing of $\gamma^{\prime}$ in Ni-base Superalloys. In: Gunter G, editor. Integral Materials Modeling: Towards Physics Based Through-Process Models. WILEY-VCH Verlag GmbH \& Co. KGaA, Weinheim, 2007. p.243.

[26] Peierls RE. The size of a dislocation. Proc. Phys. Soc. 1940;52:34.

[27] Vitek V. Intrinsic stacking faults in body-centered cubic crystals. Philosophical Magazine 1968;18:773.

[28] Khachaturyan AG. Theory of structural transformations in solids. New York: John Wiley \& Sons, 1983.

[29] Wang YU, Jin YM, Cuitino AM, Khachaturyan AG. Nanoscale phase field microelasticity theory of dislocations: model and 3D simulations. Acta Materialia 2001;49:1847.

[30] Shen C, Wang Y. Phase field model of dislocation networks. Acta Materialia 2003;51:2595.

[31] J.P. Hirth and J. Lothe, Theory of dislocations. 2nd ed. 1982, New York: Wiley.

[32] Kovarik L, Li J, Mills MJ. Manuscript in preparation. 2008.

[33] Ma A, Roters F, Raabe D. A dislocation density based constitutive law for BCC materials in crystal plasticity FEM. Computational Materials Science 2007;39:91. 